\title{
Targeting Ferroptosis Attenuates Interstitial Inflammation and Kidney Fibrosis
}

\author{
Lu Zhou $^{\mathrm{a}}$ Xian Xue ${ }^{\mathrm{b}}$ Qing Hou ${ }^{\mathrm{a}}$ Chunsun Daia, b \\ ${ }^{a}$ Center for Kidney Disease, The Second Affiliated Hospital, Nanjing Medical University, Nanjing, China; \\ ${ }^{b}$ Department of Clinical Genetics, The Second Affiliated Hospital, Nanjing Medical University, Nanjing, China
}

\section{Keywords}

Ferroptosis $\cdot$ Tubular cells $\cdot$ Kidney fibrosis

\begin{abstract}
Background: Ferroptosis, an iron-dependent form of regulated necrosis mediated by lipid peroxidation, predominantly polyunsaturated fatty acids, is involved in postischemic and toxic kidney injury. However, the role and mechanisms for tubular epithelial cell (TEC) ferroptosis in kidney fibrosis remain largely unknown. Objectives: The aim of the study was to decipher the role and mechanisms for TEC ferroptosis in kidney fibrosis. Methods: Mouse models with unilateral ureter obstruction (UUO) or ischemia/reperfusion injury (IRI) were generated. Results: We found that TEC ferroptosis exhibited as reduced glutathione peroxidase 4 (GPX4) expression and increased 4-hydroxynonenal abundance was appeared in kidneys from chronic kidney disease (CKD) patients and mouse models with UUO or IRI. Inhibition of ferroptosis could largely mitigate kidney injury, interstitial fibrosis, and inflammatory cell accumulation in mice after UUO or IRI. Additionally, treatment of TECs with (1S,3R)RSL-3, an inhibitor of GPX4, could enhance cell ferroptosis and recruit macrophages. Furthermore, inhibiting TEC fer-
\end{abstract}

(C) 2021 The Author(s)

Published by S. Karger AG, Basel

This is an Open Access article licensed under the Creative Commons Attribution-NonCommercial-4.0 International License (CC BY-NC) (http://www.karger.com/Services/OpenAccessLicense), applicable to the online version of the article only. Usage and distribution for commercial purposes requires written permission. roptosis reduced monocyte chemotactic protein 1 (MCP-1) secretion and macrophage chemotaxis. Conclusions: This study uncovers that TEC ferroptosis may promote interstitial fibrosis and inflammation, and targeting ferroptosis may shine a light on protecting against kidney fibrosis in patients with CKDs.

(c) 2021 The Author(s)

Published by S. Karger AG, Basel

\section{Introduction}

Chronic kidney disease (CKD) affects $8-16 \%$ of the population worldwide [1]. Clarifying the pathogenesis of CKD is of great significance for delaying the progression and improving the prognosis of CKDs. Kidney fibrosis is the final common pathway and the major pathological manifestation of CKD [2], which is characterized by the loss of nephron, the accumulation of extracellular matrix, activated myofibroblasts, and the infiltration of inflammatory cells [3]. Over the past decades, one significant development in renal fibrosis is a heightened appreciation for tubulointerstitial cross talk as a significant driver of progression [4-6]. As confirmed, epithelial injury remains a central event in the pathogenesis of CKD. Direct 
epithelial damage may orchestrate proinflammatory signaling [7-10]. Therefore, clarifying the pathogenesis of tubular epithelial cell (TEC) injury is of great significance for preventing the development of CKD.

Ferroptosis is a regulated cell death characterized by the iron-dependent accumulation of lipid hydroperoxides to lethal levels [11]. Emerging evidence shows the potential of triggering ferroptosis for cancer therapy, particularly for eradicating aggressive malignancies resistant to traditional therapies [12]. Ferroptosis is modulated by pharmacological perturbation of lipid repair systems involving glutathione and glutathione peroxidase 4 (GPX4) and depends on a set of positive-acting enzymatic reactions, including biosynthesis of polyunsaturated fatty acid-containing phospholipids, which are the substrates of pro-ferroptosis lipid peroxidation products, by ACSL4 and LPCAT3, and selective oxygenation of polyunsaturated fatty acid-phosphatidylethanolamines by lipoxygenases. TECs require an ample energy supply for reabsorption in the kidney, so fatty acid oxidation is the preferred energy source. Typically, fatty acid uptake, oxidation, and synthesis are tightly balanced to avoid intracellular lipid accumulation. Enabled by the first described GPX4 inhibitor, (1S,3R)-RSL-3 (RSL3), as well as mice with tamoxifen-inducible whole-body deletion of Gpx4 (except in brain), unequivocal independent evidence was obtained, showing not only that GPX4 is a tempting drug target for driving cancer cell death, but also that kidney tubular cells are perhaps the most ferroptosis-sensitive cells in mammals, respectively. It has been demonstrated that ferroptosis is an integral process in the pathogenesis of cisplatin-induced acute kidney injury (AKI) [13] and mediates postischemic renal necrosis [14]. However, its relevance to the process of fibrosis remains to be investigated.

This study uncovered the appearance of tubular cell ferroptosis in kidneys from patients with CKD and mouse models with unilateral ureter obstruction (UUO) or ischemia/reperfusion injury (IRI) nephropathy. Inhibition of ferroptosis significantly reduced inflammatory cell accumulation and kidney fibrosis in both UUO and IRI mice. Overall, our results demonstrate that targeting ferroptosis attenuates interstitial inflammation and kidney fibrosis.

\section{Materials and Methods}

Mice and Animal Models

Male CD1 mice weighing approximately 18-20 g were acquired from the specific Pathogen-Free Laboratory Animal Center of Nanjing Medical University. Mice were performed with left renal
UUO or left renal IRI. For UUO experiments, the animals were divided into 2 groups: (1) mice were injected with normal saline; (2) mice were injected with ferrostatin-1 (Fer-1) (5 mg/kg/day) or deferoxamine (DFO) $(100 \mathrm{mg} / \mathrm{kg} /$ day $)$ from 3 days before to 10 days after surgery. For IRI experiments, mice were divided into 2 groups: (1) mice were injected with normal saline; (2) mice were injected with Fer-1 ( $5 \mathrm{mg} / \mathrm{kg} /$ day) or DFO (100 mg/kg/day) 7 days after surgery until 21st day. Fer-1 (cat.: SML0583; Sigma) was prepared in a 5\% DMSO solution and injected intraperitoneally once a day. DFO (cat.: D9533; Sigma) was prepared in phosphate-buffered saline and injected intraperitoneally twice a day at 9 a.m. and 9 p.m. The mice were euthanized on day 10 after UUO and day 21 after IRI. The UUO and IRI kidneys, as well as the contralateral kidneys, were removed. One portion of the kidney was fixed in $10 \%$ phosphate-buffered formalin, followed by paraffin embedding for histological and immunohistochemical staining. Another portion was immediately frozen in Tissue-Tek optimum cutting temperature compound (Sakura Finetek, Torrance, CA, USA) for cryosection. The remaining kidney tissue was snap-frozen in liquid nitrogen and stored at $-80^{\circ} \mathrm{C}$ for extraction of RNA and protein.

\section{Cell Culture and Treatment}

RAW264.7 and normal rat kidney tubular epithelial cells (NRK-52E) were ordered from ATCC (Manassas, VA, USA). RAW264.7 cells were cultured in Dulbecco's modified Eagle's medium supplemented with $10 \%$ fetal bovine serum (Invitrogen, Grand Island, NY, USA). NRK-52E cells were cultured in Dulbecco's modified Eagle's medium/F12 medium supplemented with $5 \%$ newborn calf serum (Invitrogen, Grand Island, NY, USA). RSL3 (cat.: S8155; Selleck) were added to NRK-52E at the concentration of $1 \mu \mathrm{M}$ for $4 \mathrm{~h}$. Fer- 1 was added to NRK-52E 30 min before RSL3 at the concentration of $2 \mu \mathrm{M}$. MCP-1 and scramble siRNA (GenePharma, Shanghai, China) were transfected into NRK-52E cells using Lipofectamine 3000 reagent (Invitrogen) according to the manufacturer's instruction.

\section{Coculture and Migration Assays}

We cocultured RAW264.7 and NRK-52E cells using Transwell chambers with $8-\mu \mathrm{m}$ pores on the membrane (cat.: MCEP24H48; Millicell) in a 24 -well plate. NRK-52E cells were cultured in the lower compartments of the Transwell chamber. After $4 \mathrm{~h}$ of treatment with RSL3, NRK-52E cells were washed with essential medium 3 times. Then, we seeded RAW264.7 cells at a density of 100,000 cells in $0.5 \mathrm{~mL}$ medium to the upper compartments of the Transwell chamber, coculture with NRK-52E cells for $10 \mathrm{~h}$. The noninvasive RAW264.7 cells in the upper chambers were removed by cotton swabs and stained with a $0.5 \%$ crystal violet solution for $20 \mathrm{~min}$. We counted the migratory or invasive cells under an optical microscope and took the average of 5 high-power fields.

\section{Histology and Immunohistochemistry}

Kidney samples were fixed in $10 \%$ neutral formalin and embedded in paraffin. We stained slices at $3 \mu \mathrm{m}$ thickness with periodic acid-Schiff, Masson, and Sirius red. The control group of human kidney tissues was the normal tissue surrounding kidney neoplasms, obtained from 5 patients who underwent nephrectomy due to malignancies. We obtained human CKD kidney biopsies from patients diagnosed with IgA nephropathy, chronic interstitial nephritis, or diabetic nephropathy in the Second Affiliated 
Hospital of Nanjing Medical University. The Institutional Review Board at the Second Affiliated Hospital approved all studies involving human tissues.

For immunohistochemical staining, kidney sections were deparaffinized, hydrated, and antigen-retrieved. Endogenous peroxidase activity was quenched by $3 \% \mathrm{H}_{2} \mathrm{O}_{2}$. Tissue sections were then blocked with $5 \%$ normal donkey serum, followed by incubation with anti-GPX4 (cat.: 125066; Abcam), anti-4-hydroxynonenal (HNE) (cat.: 46545; Abcam), and anti-MCP-1 (cat.: 66272-1-lg; Proteintech) overnight at $4^{\circ} \mathrm{C}$. After incubation with secondary antibody for $30 \mathrm{~min}$, sections were incubated with $\mathrm{DAB}$ reagents for coloration (cat.: ZLI-9018; ZSGB-BIO) and then restained with hematoxylin. Slides were viewed with a Nikon Eclipse 80i Epifluorescence microscope equipped with a digital camera (DS-Ril; Nikon).

\section{Semiquantitative Analysis of Immunohistochemistry}

We used a semiquantitative analysis of immunohistochemistry to determine the relative abundance of protein expression - a total score based on staining intensity combined with the percentage of positive renal tubules. The staining intensity is scored based on the staining characteristics of most tubules (the depth of staining needs to be compared with the background coloring): 0 points for no staining, 1 point for light yellow, 2 points for brownish yellow, and 3 points for tan. The number of renal tubules in each score was counted then. The final score of each selected field was calculated according to the formula: score $=(0 \times$ number of renal tubules with 0 points $+1 \times$ number of renal tubules with 1 point $+2 \times$ number of renal tubules with 2 points $+3 \times$ number of renal tubules with 3 points)/total number of observed renal tubules. We evaluated at least 5 randomly selected fields under a $400 \times$ microscope for each kidney section and calculated an average score.

\section{Kidney Injury Score}

Paraffin-embedded kidney sections (3 $\mu \mathrm{m}$ thickness) were stained with periodic acid-Schiff. A semiquantitative scoring method was used to define kidney injury by losing brush border, tubular cell necrosis, and cellular casts. Score 0 represents injury area $<10 \%$, whereas scores $1,2,3$, and 4 represent the injury involving $10-25,25-50,50-75$, and $>75 \%$ of the kidney tissue area, respectively. We evaluated at least 5 randomly selected fields under a $400 \times$ microscope for each kidney section and calculated an average score.

\section{Immunofluorescent Staining}

Kidney cryosections at $3 \mu \mathrm{m}$ thickness were fixed for $15 \mathrm{~min}$ in $4 \%$ paraformaldehyde, followed by permeabilization with $0.5 \%$ Triton X-100 in phosphate-buffered saline for $5 \mathrm{~min}$ at room temperature. After blocking with $2 \%$ donkey serum for $60 \mathrm{~min}$, the slides were immunostained with anti-fibronectin (FN) (cat.: F3648; Sigma-Aldrich, St. Louis, MO, USA), anti-a-SMA (a-smooth muscle actin) (cat.: A5228; Sigma-Aldrich), anti-F4/80 (cat.: 14-4801; eBioscience, San Diego, CA, USA), and anti-CD3 (cat.: 555273; BD Pharmingen), followed by staining with fluorescein isothiocyanate or tetramethylrhodamine-conjugated secondary antibody.

\section{TUNEL Assay}

Dead cells were detected by the DeadEnd ${ }^{\mathrm{TM}}$ Fluorometric TUNEL System (cat.: G3250; Promega) according to the instruction.
Measurement of Total Collagen in Kidney Tissue

Tissue paraffin sections at $3 \mu \mathrm{m}$ thickness were dried at $55^{\circ} \mathrm{C}$ for $1 \mathrm{~h}$, followed by xylene, xylene-ethanol (1:1), ethanol, ethanolpure water $(1: 1)$, and pure water dewaxing and hydration. The mixed dye $(0.1 \%$ Sirius red F3BA $+0.1 \%$ Fast Green FCF [SigmaAldrich]) was soaked overnight at room temperature and decolorized with $0.1 \mathrm{~N} \mathrm{NaOH}$ and methanol solution (1:1). Then, the OD values at 540 and $605 \mathrm{~nm}$ were read by a spectrophotometer and the contents of total collagen and protein in the tissue sections were calculated according to the formula: Collagen content $(\mu \mathrm{g} /$ tissue section $)=(\mathrm{OD} 540-[\mathrm{OD} \times 605 \times 0.291]) / 37.3 \times 1,000$; protein content $(\mu \mathrm{g} /$ tissue section $)=$ OD605/1.96 $\times 1,000$. We used the total collagen/protein content to reflect the collagen content in the tissue.

\section{Quantitative Real-Time Polymerase Chain Reaction}

Total RNA was extracted using a Trizol reagent (Invitrogen) according to the manufacturer's instructions. cDNA was synthesized with $1 \mu \mathrm{g}$ of total RNA, ReverTra Ace (Vazyme, Nanjing, China), and oligo (dT) 12-18 primers. Gene expression was measured by a real-time polymerase chain reaction assay (Vazyme) and 7300 real-time polymerase chain reaction systems (Applied Biosystems, Foster City, CA, USA). The relative amount of mRNA or gene to internal control was calculated using Equation (2) $\triangle \mathrm{CT}$, in which $\triangle \mathrm{CT}=\mathrm{CT}$ gene $-\mathrm{CT}$ control.

\section{Western Blotting Analysis}

The kidneys were lysed with a radioimmunoprecipitation assay solution containing $1 \%$ NP40, $0.1 \%$ sodium dodecyl sulfate, 100 $\mathrm{mg} / \mathrm{mL}$ phenylmethanesulfonyl fluoride, $1 \%$ protease inhibitor cocktail, and 1\% phosphatase I and II inhibitor cocktail (Sigma, St. Louis, MO, USA) on ice. The supernatants were collected after centrifugation at $13,000 \mathrm{~g}$ at $4^{\circ} \mathrm{C}$ for $30 \mathrm{~min}$. According to the manufacturer's instructions, protein concentration was determined by the bicinchoninic acid protein assay kit (Pierce Thermo-Scientific, Rockford, IL, USA). An equal amount of protein was loaded into 10 or $12 \%$ sodium dodecyl sulfate-polyacrylamide gel electrophoresis and transferred onto nitrocellulose filter membranes. The primary antibodies were anti-FN, anti- $\alpha$-SMA, anti-4-HNE, antiGPX4, and anti-GAPDH (cat.: FL-335; Santa Cruz Biotechnology, Dallas, TX, USA), and anti-tubulin (cat.: T9026; Sigma). Quantification was performed by measuring the intensity of the signals with the National Institutes of Health ImageJ software package.

\section{Statistical Analyses}

Data were expressed as mean \pm SEM. Statistical analysis of data was performed using GraphPad Prism 6 (GraphPad Software, San Diego, CA, USA). A comparison between groups was made using a 1-way analysis of variance, followed by the Student-Newman-Keuls test. $p<0.05$ was considered statistically significant.

\section{Results}

\section{Ferroptosis Is Triggered in Tubular Cells of CKD}

Patients and Mice with UUO or IRI Nephropathy

To examine the role of ferroptosis in kidney fibrosis, we first performed immunohistochemical staining for 


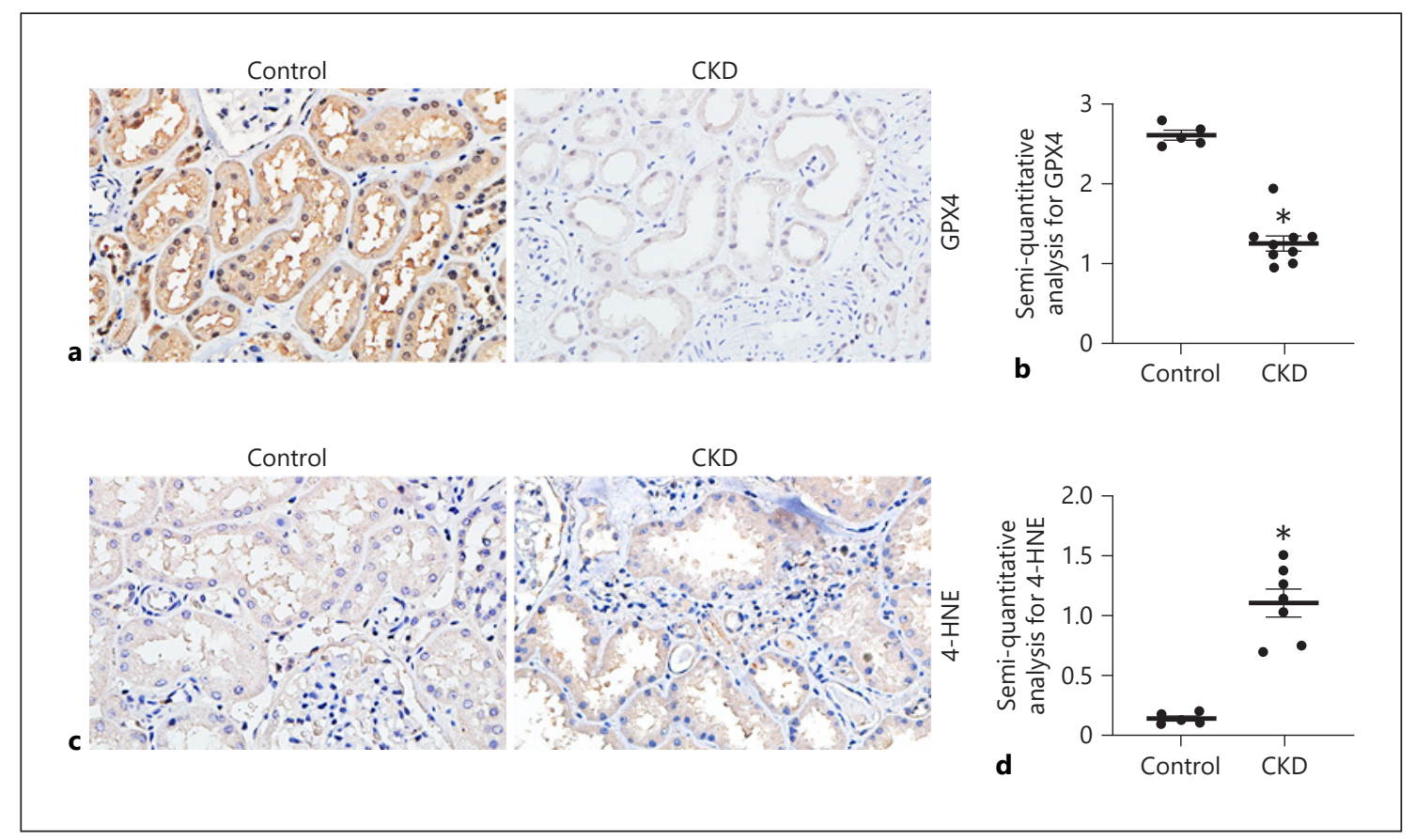

Fig. 1. Repression of GPX4 and induction of 4-HNE in renal tubules of patients with CKDs. a, c Representative micrographs of immune staining of GPX4 and 4-HNE in human kidney tissues. Scale bar, $20 \mu \mathrm{m}$. b, d Semiquantitative analysis showing GPX4 and 4-HNE expression in the kidney of CKD patients. ${ }^{*} p<0.05$ versus control. GPX4, glutathione peroxidase 4; 4-HNE, 4-hydroxynonenal; CKD, chronic kidney disease.

GPX4 and 4-HNE on kidney biopsies from CKD patients. The adjacent kidney tissues of kidney tumors from patients were used as controls. The results showed that the expression of GPX4 was high in control kidney tissues, and 4-HNE, one of the end products of lipid peroxidation, was rarely detected, while in renal tubules of CKD patients, GPX4 abundance was significantly decreased, and 4-HNE was largely induced (shown in Fig. 1).

We next examined the abundance of GPX 4 and 4-HNE in mouse kidneys after UUO or IRI. Western blotting analyses revealed that GPX4 abundance was decreased mainly in a time-dependent manner in kidneys after UUO (shown in Fig. 2a). GPX4 was also decreased in kidneys after IRI (shown in Fig. 2b). Immunohistochemical staining indicated that GPX4 was primarily expressed in TECs in control mice, whereas it was largely decreased in UUO or IRI kidneys (shown in Fig. 2c, d). However, immunohistochemical staining revealed that 4-HNE was increased in TECs in both UUO and IRI kidneys (shown in Fig. 2c-f). These results suggest that lipid peroxidation and ferroptosis increased in the tubules of fibrotic kidneys.

\section{Inhibition of Ferroptosis Reduces Extracellular Matrix} Deposition in the Fibrotic Kidneys

To further study the role of ferroptosis in renal fibrosis, we injected CD1 male mice with Fer-1 ( $5 \mathrm{mg} / \mathrm{kg} /$ day), a specific inhibitor of ferroptosis, intraperitoneally at 3 days before UUO surgery until the 10th day of UUO and 7 days after IRI surgery until the 21st day of IRI (shown in Fig. 3a). In the control groups, the kidney morphology was comparable between the vehicle-treated and Fer1-treated, while in the UUO kidneys from Fer-1-treated mice, tubular atrophy and interstitial fibrosis were attenuated compared to those from vehicle-treated mice (shown in Fig. 3b-e). Similarly, in the fibrotic kidneys after IRI from the Fer-1-treated, kidney injury, interstitial fibrotic area, and total collagen deposition were all significantly diminished compared to those from vehicletreated mice (shown in Fig. 3f-i). Also, we used another ferroptosis inhibitor - DFO - an iron chelator to treat the mice with UUO or IRI nephropathy. Similarly, DFO treatment significantly reduced kidney injury and fibrosis caused by UUO or IRI (shown in Fig. 4).

In addition, the Western blotting analysis showed that Fer-1- or DFO-treated mice with UUO or IRI had less abundance of FN and $\alpha$-SMA compared to vehicle-treat- 


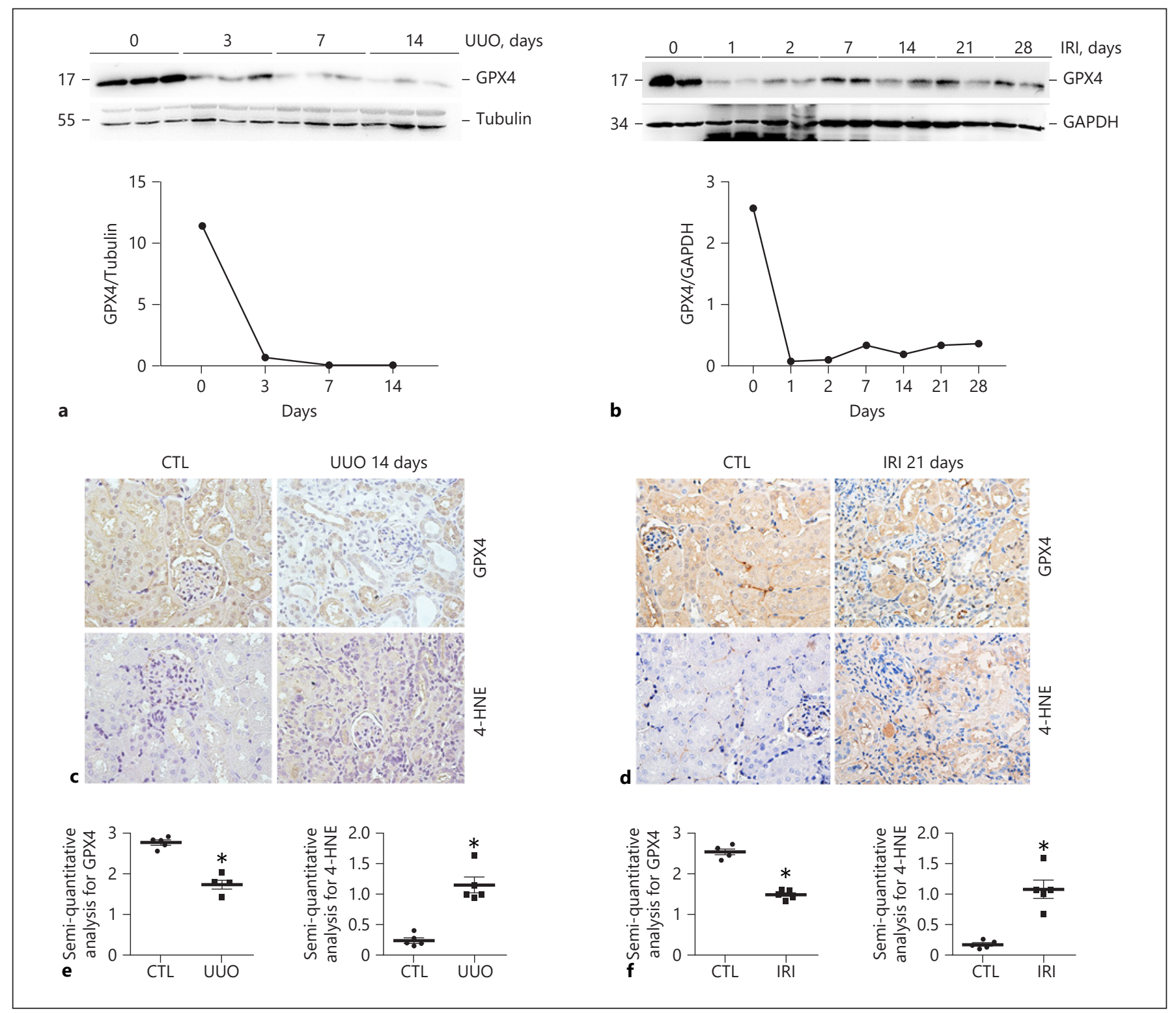

Fig. 2. Repression of GPX4 and induction of 4-HNE in the mouse kidneys. a, b Western blotting analysis and semiquantitative analysis showing GPX4 expression in kidney tissues after UUO or IRI. c, $\mathbf{d}$ Immune staining showing the repression of GPX4 and induction of 4-HNE in kidney tubules after UUO or IRI. Scale bar,

ed mice (shown in Figs. 5a-f and 6a-f). Decreased expression of FN and $\alpha$-SMA was further confirmed by immunofluorescence staining (shown in Figs. 5g, h, 6g, h).

As one type of regulated necrosis, inhibition of ferroptosis might lead to reduced cell death, so we detected tubular cell death after Fer-1 or DFO treatment by TUNEL staining. The results show that TUNEL ${ }^{+}$TECs have vastly decreased with Fer-1 or DFO treatment after UUO or
$20 \mu \mathrm{m}$. e, f Semiquantitative analysis showing GPX4 and 4-HNE expression in kidney tubules after UUO or IRI. ${ }^{*} p<0.05$ versus sham control. GPX4, glutathione peroxidase 4; CKD, chronic kidney disease; UUO, unilateral ureter obstruction; IRI, ischemia/reperfusion injury; 4-HNE, 4-hydroxynonenal.

IRI (shown in Fig. 7). Taken together, these results indicate that inhibition of ferroptosis attenuated kidney injury and fibrosis in mice with UUO and IRI nephropathy.

\section{Fer-1 Attenuates Inflammation in the Fibrotic \\ Kidneys}

To assess the inflammatory cell accumulation in the kidneys, we stained kidney tissues with antibodies against 


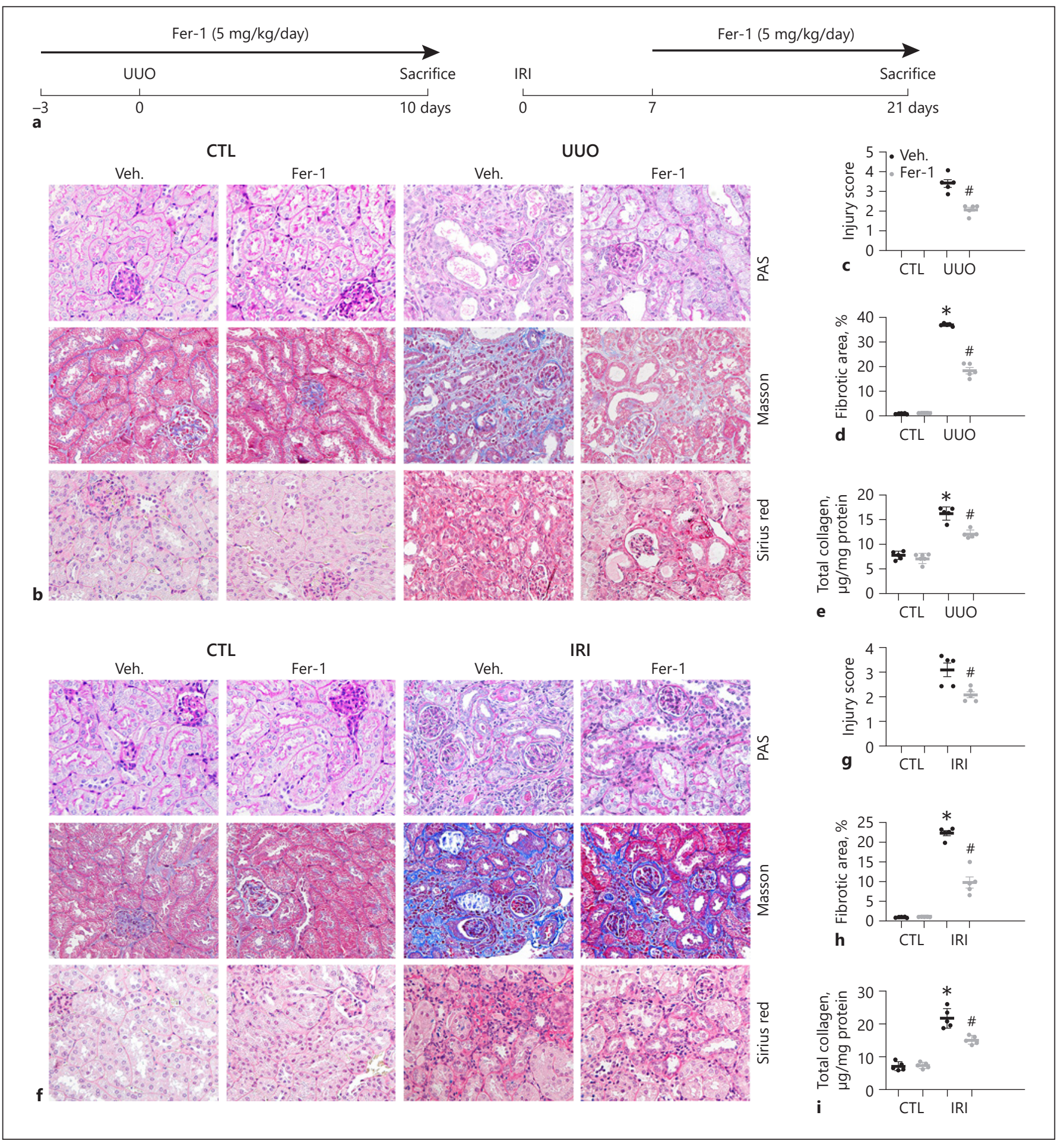

Fig. 3. Inhibition of ferroptosis by Fer-1 ameliorates kidney fibrosis in mice. a Summary of experimental strategy. b, f Representative micrographs for PAS, Masson, and Sirius red staining in kidney tissues. Scale bar, $20 \mu \mathrm{m}$. c, g Injury Score showing the degree of kidney injury among groups as indicated. ${ }^{\#} p<0.05$ versus vehicle-treated mice, $n=5$. $\mathbf{d}$, $\mathbf{h}$ Semiquantitative analysis of Masson staining showing the fibrotic area in kidney tissues among groups as indicated. ${ }^{*} p<0.05$ versus sham control; ${ }^{*} p<0.05$ versus vehicle-treated mice with UUO or IRI, $n=5$. e, i Quantitative analysis of collagen content in renal tissues of different groups. ${ }^{*} p<0.05$ versus sham control; ${ }^{*} p<0.05$ versus vehicle-treated mice after UUO or IRI, $n=5$. UUO, unilateral ureter obstruction; IRI, ischemia/reperfusion injury; Fer-1, ferrostatin-1; PAS, periodic acidSchiff. 


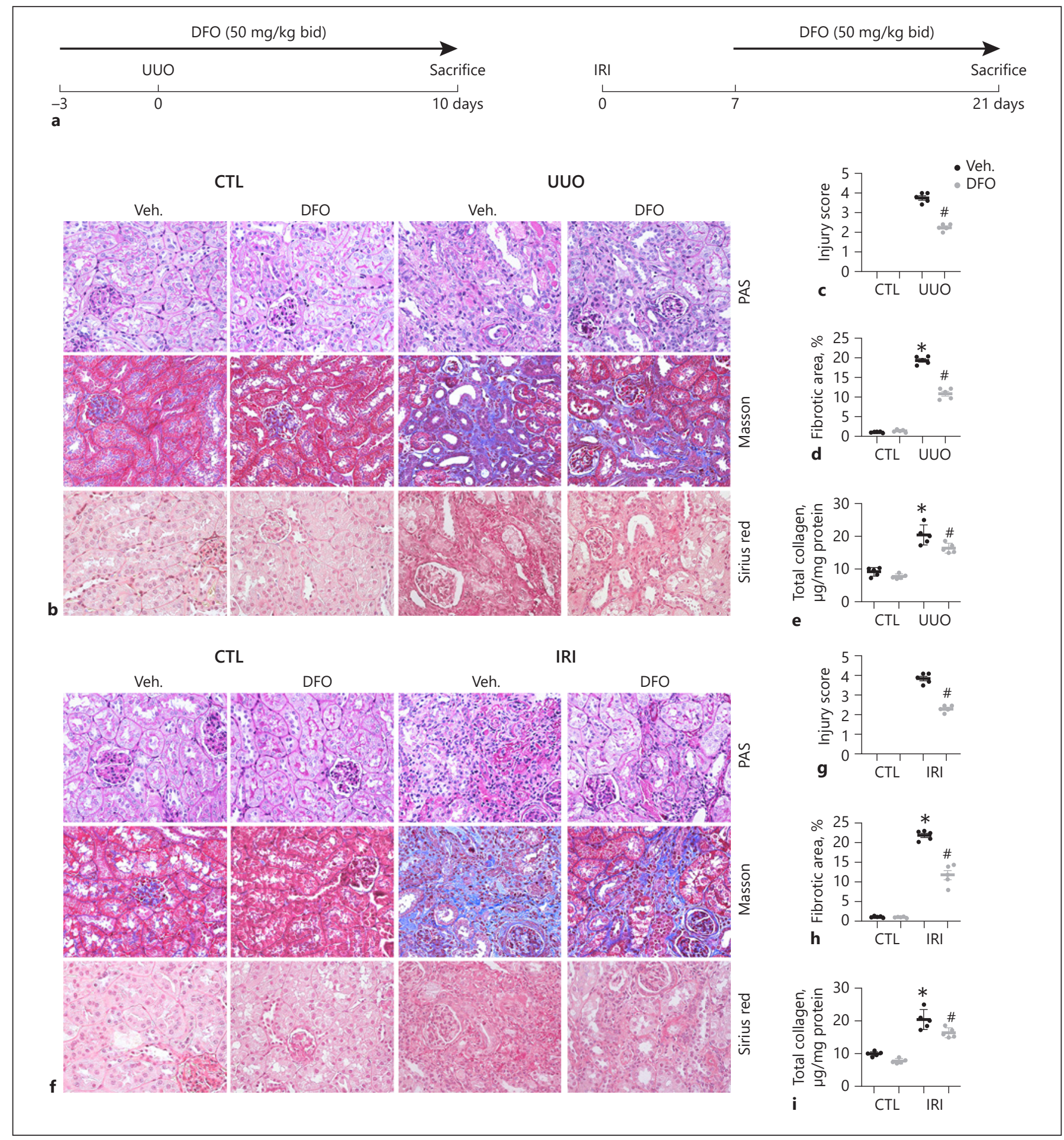

Fig. 4. Inhibition of ferroptosis by DFO attenuates kidney fibrosis in mice. a Experimental strategy. $\mathbf{b}, \mathbf{f}$ Representative micrographs for PAS, Masson, and Sirius red staining in kidney tissues. Scale bar, $20 \mu \mathrm{m}$. c, $\mathbf{g}$ Injury Score showing the degree of kidney injury among groups as indicated. ${ }^{\#} p<0.05$ versus vehicle-treated mice, $n=5$. $\mathbf{d}, \mathbf{h}$ Semiquantitative analysis of Masson staining showing the fibrotic area in kidney tissues among groups as indicated. ${ }^{*} p<$
0.05 versus sham control; ${ }^{\#} p<0.05$ versus vehicle-treated mice with UUO or IRI, $n=5$. e, i Quantitative analysis of collagen content in renal tissues of different groups. ${ }^{*} p<0.05$ versus sham control; ${ }^{*} p<0.05$ versus vehicle-treated mice with UUO or IRI, $n=5$. UUO, unilateral ureter obstruction; IRI, ischemia/reperfusion injury; DFO, deferoxamine; PAS, periodic acid-Schiff. 


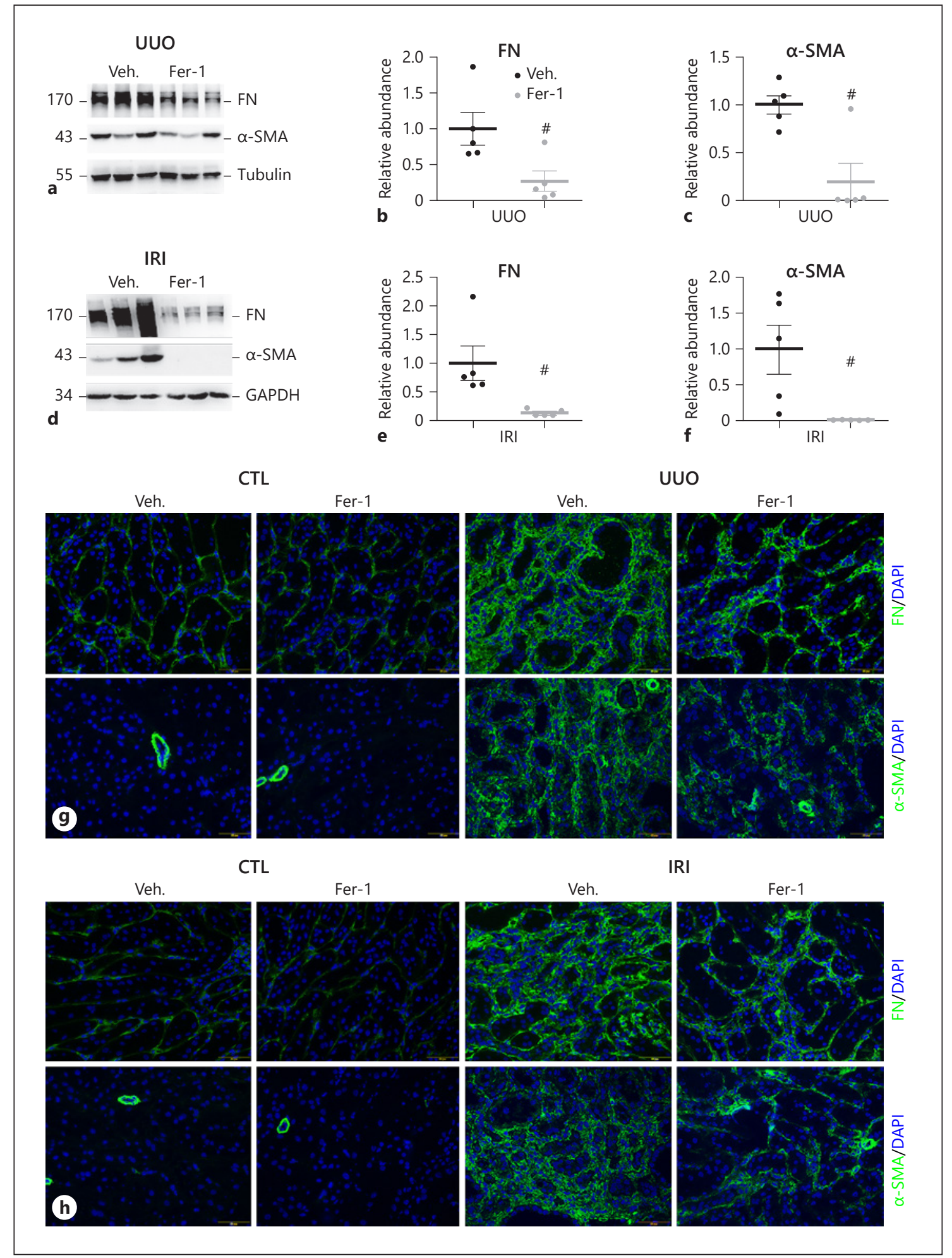

Fig. 5. Inhibition of ferroptosis by Fer-1 reduces FN and $\alpha$-SMA expression in mice. a, $\mathbf{d}$ Western blotting analyses for FN and $\alpha$-SMA in kidney tissues from different groups as indicated. b, c, e, f Quantitative analysis for FN and a-SMA in UUO and IRI kidneys from different groups. ${ }^{\#} p<0.05$ versus vehicle-treated mice with UUO or
IRI, $n=5 . \mathbf{g}, \mathbf{h}$ Representative micrographs of immunofluorescence staining showing FN and $\alpha$-SMA-positive area in kidney tissues among groups as indicated. Scale bar, $20 \mu \mathrm{m}$. UUO, unilateral ureter obstruction; IRI, ischemia/reperfusion injury; Fer-1, ferrostatin-1; FN, fibronectin; $\alpha$-SMA, $\alpha$-smooth muscle actin. 


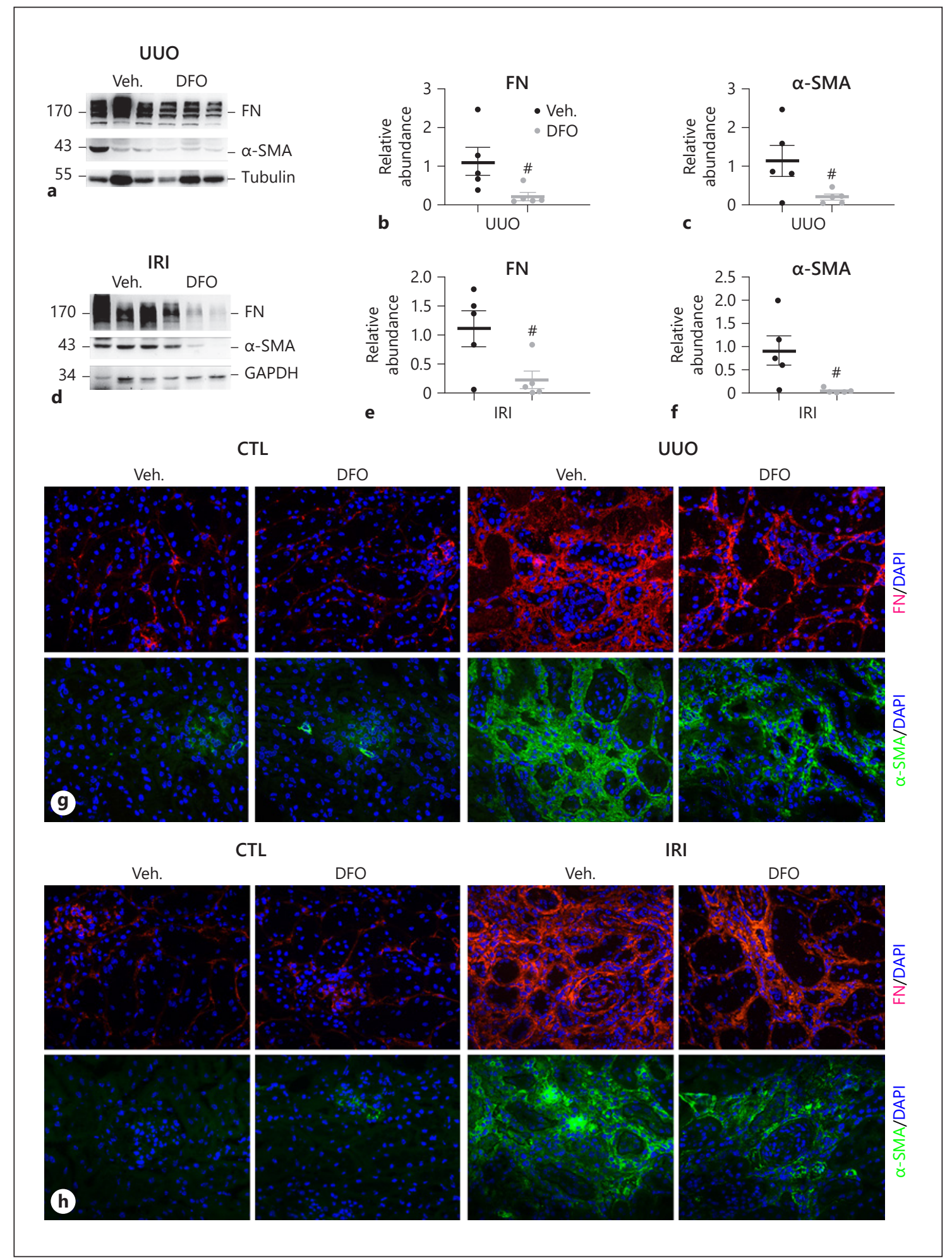

Fig. 6. Inhibition of ferroptosis by DFO reduces FN and $\alpha$-SMA expression in mice. a, $\mathbf{d}$ Western blotting analyses for FN and $\alpha$-SMA in kidney tissues from different groups as indicated. b, c, e, f Quantitative analysis for FN and a-SMA in UUO and IRI kidneys from different groups. ${ }^{\#} p<0.05$ versus vehicle-treated mice with UUO or
IRI, $n=5 . \mathbf{g}, \mathbf{h}$ Representative micrographs of immunofluorescence staining showing FN and $\alpha$-SMA-positive area in kidney tissues among groups as indicated. Scale bar, $20 \mu \mathrm{m}$. UUO, unilateral ureter obstruction; IRI, ischemia/reperfusion injury; DFO, deferoxamine; FN, fibronectin; $\alpha$-SMA, $\alpha$-smooth muscle actin. 
Fig. 7. Inhibition of ferroptosis reduces tubule epithelial cell death in mouse kidneys. a Representative images of TUNEL staining showing dead cells in kidney tissues with or without Fer-1 treatment after UUO or IRI. Scale bar, $20 \mu \mathrm{m}$. b Quantitative analysis of TUNEL ${ }^{+}$TECs. ${ }^{*} p<0.05$ versus vehicle-treated mice, $n=5$. c Representative images of TUNEL staining showing dead cells in kidney tissues with or without DFO treatment after UUO or IRI. Scale bar, $20 \mu \mathrm{m}$. d Quantitative analysis of TUNEL ${ }^{+}$TECs. ${ }^{*} p<0.05$ versus vehicletreated mice, $n=5$. UUO, unilateral ureter obstruction; IRI, ischemia/reperfusion injury; Fer-1, ferrostatin-1; DFO, deferoxamine; TECs, tubular epithelial cells.
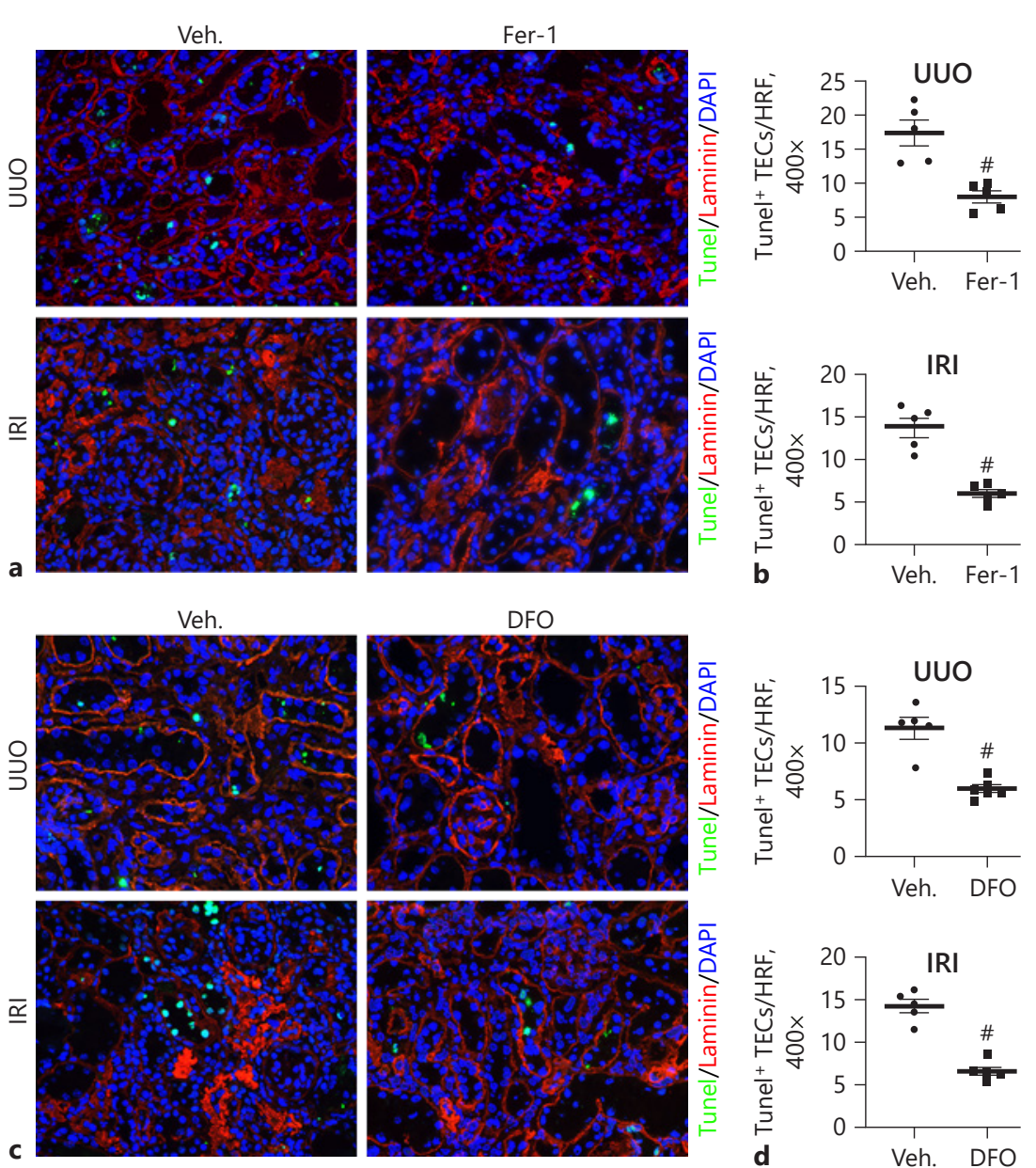

F4/80 and CD3 to identify macrophages and T lymphocytes, respectively. In the UUO kidneys from the Fer1-treated mice, macrophage and $\mathrm{T}$ lymphocyte accumulation were much less than that treated with vehicle (shown in Fig. 8a, c, d). Similarly, macrophage accumulation and $\mathrm{T}$ lymphocyte infiltration were significantly decreased by treatment with Fer-1 in the fibrotic kidneys at 3 weeks after IRI (shown in Fig. 8b, e, f).

We then co-stained kidney tissues with antibodies against 4 -HNE and F4/80. In both UUO and IRI kidneys, macrophages were accumulated around 4-HNE-positive staining areas, indicating that ferroptotic TECs might recruit macrophages (shown in Fig. 8g). These results suggest that inhibition of TEC ferroptosis reduced the accumulation of macrophage and $\mathrm{T}$ lymphocytes within the fibrotic kidneys.
Inhibition of Tubular Cell Ferroptosis Reduces

Macrophage Chemotaxis through Downregulating MCP-1 Production

To further confirm that ferroptotic TEC chemotaxis macrophages, we performed a Boyden chamber transwell assay in which RAW264.7 cells were cocultured with NRK-52E cells treated with vehicle or RSL3, a stimulator of ferroptosis (shown in Fig. 9a). Much more RAW264.7 cells cultured over RSL3-treated NRK-52E cells for $4 \mathrm{~h}$ migrated through the porous membrane within $10 \mathrm{~h}$, compared to that of cells cultured over vehicle-treated NRK-52E cells (shown in Fig. 9b, c).

To further explore the mechanism of tubular cell ferroptosis in recruiting macrophages, we detected mRNA abundance of MCP-1, one of the key chemokines that regulate migration and infiltration of monocytes/macrophages, in RSL3-treated NRK-52E cells. Obviously, MCP- 


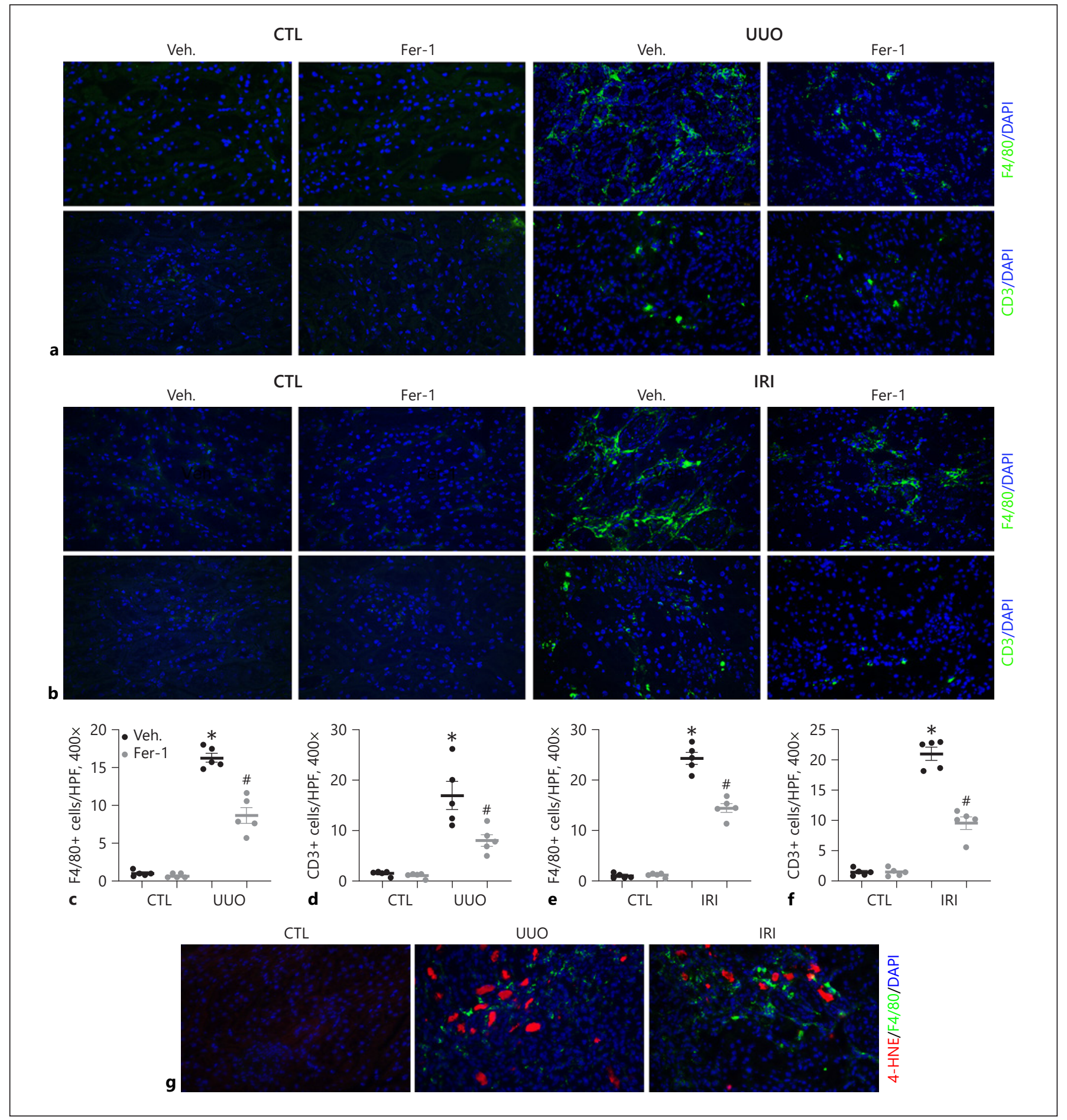

Fig. 8. Fer-1 treatment alleviates inflammatory cell accumulation in the fibrotic kidneys. a, b Representative micrographs of immune staining for F4/80 and CD3 in kidney tissues. Scale bar, $20 \mu \mathrm{m}$. c, e Graphs showing quantitative analysis of F4/80-positive cells in kidney tissues from the Fer- 1 treatment and control littermates after UUO or IRI. Scale bar, $20 \mu \mathrm{m} .{ }^{*} p<0.05$ versus sham control; ${ }^{\#} p<0.05$ versus vehicle-treated mice after UUO or IRI, $n=5$. $\mathbf{d}$, $\mathbf{f}$ Graphs showing quantitative analysis of CD3-positive cells in kidney tissues from the Fer-1 treatment and control littermates after UUO or IRI. Scale bar, $20 \mu \mathrm{m} .{ }^{*} p<0.05$ versus sham control; ${ }^{*} p<0.05$ versus vehicle-treated mice after UUO or IRI, $n=5$. $g$ Representative micrographs of co-staining for F4/80 and 4 -HNE in kidney tissues after UUO or IRI. Scale bar, $20 \mu \mathrm{m}$. UUO, unilateral ureter obstruction; IRI, ischemia/reperfusion injury; 4-HNE, 4-hydroxynonenal; Fer-1, ferrostatin-1; 4-HNE, 4-hydroxynonenal. 


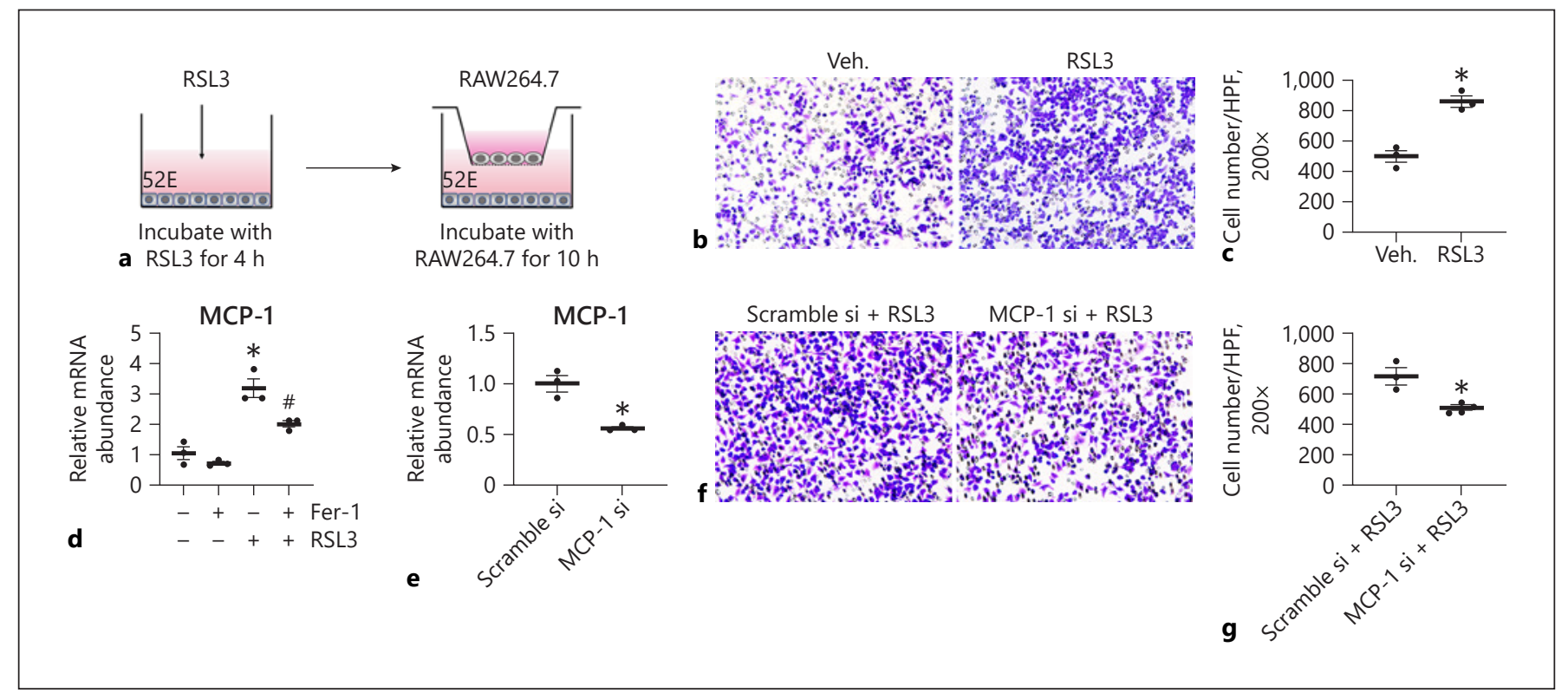

Fig. 9. Induction of NRK-52E cell ferroptosis facilitates macrophage migration. a Experimental design for RAW264.7 cells migration assay. b Representative images for Boyden chamber migration assay in cultured RAW264.7 cells. RAW264.7 cells were treated with vehicle or RSL3 $(1 \mu \mathrm{M})$ for $4 \mathrm{~h}$. c Quantitative analysis showing the number of migrated cells in the lower chamber with crystal violet solution $(0.1 \%) .{ }^{*} p<0.05$ versus vehicle. $\mathbf{d}$ qRT-PCR analysis showing the mRNA abundance for MCP-1 in NRK-52E after vehicle or RSL3 stimulation with or without Fer-1 treatment. ${ }^{*} p<0.05$ versus vehicle control; ${ }^{\#} p<0.05$ versus vehicle-treated NRK-52E after RSL3 stimulation. e qRT-PCR analysis demon- strating the downregulation of MCP-1 after siRNA transfection. ${ }^{*} p<0.05$ versus scramble control. $\mathbf{f}$ Representative images for Boyden chamber migration assay in cultured RAW264.7 cells. RAW264.7 cells were pretreated with scramble or MCP-1 siRNA for $24 \mathrm{~h}$. $g$ Quantitative analysis showing the number of migrated cells in the lower chamber with crystal violet solution $(0.1 \%)$. ${ }^{*} p<$ 0.05 versus scramble-treated. Fer-1, ferrostatin-1; RSL3, (1S,3R)RSL-3; qRT-PCR, quantitative real-time polymerase chain reaction; MCP-1, monocyte chemotactic protein 1; NRK-52E, normal rat kidney tubular epithelial cells.
Fig. 10. Inhibition of ferroptosis reduces MCP-1 expression in the fibrotic kidneys. a, c qRT-PCR analysis showing the mRNA abundance for MCP-1 in kidney tissues after UUO or IRI with or without Fer-1 treatment. ${ }^{*} p<0.05$ versus sham control; ${ }^{*} p<$ 0.05 versus vehicle-treated mice after UUO or IRI, $n=4$. b, d Graphic presentation showing the immune staining for MCP-1 in kidney tissues among groups as indicated. Scale bar, $20 \mu \mathrm{m}$. qRT-PCR, quantitative real-time polymerase chain reaction; UUO, unilateral ureter obstruction; IRI, ischemia/reperfusion injury; Fer-1, ferrostatin-1; MCP-1, monocyte chemotactic protein 1.

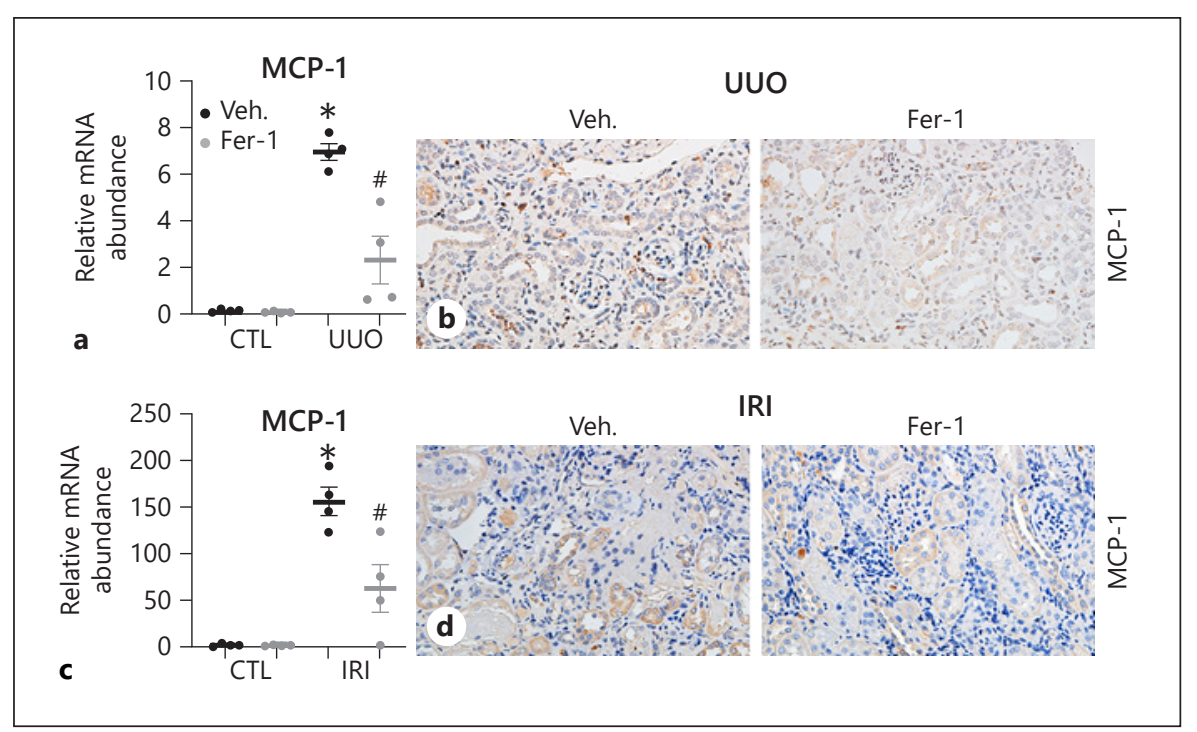


1 was significantly induced in RSL3-treated cells compared with vehicle-treated cells, whereas this change was reversed by Fer-1 treatment (shown in Fig. 9d). Less RAW264.7 cells cultured over RSL3-treated NRK-52E cells transfected with MCP-1 siRNA migrated through the porous membrane than that of cells cultured over scramble transfected cells (shown in Fig. 9f, g). Consistently, MCP-1 mRNA abundance was higher in UUO kidneys than in control kidneys, and it was largely inhibited by Fer1 treatment (shown in Fig. 10a). These results were similar in IRI mice, suggesting that ferroptotic TECs may release MCP-1 that in turn recruits macrophages (shown in Fig. 10c). Concomitantly, immune staining for MCP-1 in kidney tissues confirmed the induction of MCP-1 in the injured tubules after UUO or IRI, which was abolished by Fer-1 treatment (shown in Fig. 10b, d). These results suggest that inhibition of TEC ferroptosis reduces macrophage chemotaxis through decreasing MCP-1 production.

\section{Discussion}

Recently, it has been demonstrated that treating hepatocyte-specific transferrin knockout mice with the ferroptosis inhibitor Fer-1 potently rescued liver fibrosis induced by either high dietary iron or carbon tetrachloride $\left(\mathrm{CCl}_{4}\right)$ injection [15]. In this study, we demonstrated that treating UUO or IRI mice with Fer-1 or DFO mitigated kidney fibrosis. First, we found increased 4-HNE and decreased the expression of GPX4 in kidney biopsies of patients with CKD and mouse models of fibrotic kidney disease, suggesting that ferroptosis occurs. Second, inhibition of ferroptosis significantly reduced the expression of FN and $\alpha$-SMA and inflammatory cell infiltration. Third, inhibiting ferroptosis by Fer-1 could markedly reduce MCP-1 secretion by TECs, which attribute to decreased macrophage chemotaxis. To the best of our knowledge, this is the first report that demonstrated the crucial role of ferroptosis of TECs in kidney fibrosis of CKD.

Renal tubules and tubulointerstitium make up a significant portion of the kidney and are the major sites in response to injuries, including hypoxia, proteinuria, toxins, metabolic disorders, and senescence [16]. The innate immune characteristics demonstrate that TECs are the immune responders to a wide range of insults, with the consequent production and release of bioactive molecules that drive interstitial inflammation and fibrosis [17]. Many studies suggest that maladaptive repair of TECs leads to injured TECs being transformed into a secretory phenotype, and elicit proinflammatory (e.g., interleukin, tumor necrosis factor, chemokines, adhesion molecules, reactive oxygen species, and growth factors) [18-20] and profibrotic mediators (e.g., TGF $\beta$, CTGF, Wnt, Notch, RAS components, and complement) [21, 22].

Under the stimulation of injury, renal tubular cell death may occur by regulated necrosis (RN) or apoptosis. Depending on the stimulus, apoptosis and necrosis could lie on a continuum of cell death, so the 2 forms are not mutually exclusive and can coexist in many pathological conditions. Plenty of studies have demonstrated the pathogenic role of tubular cell apoptosis and necroptosis in kidney injury. Ferroptosis - a previously unknown form of controlled cell death with significant similarities to oxytosis - was described in 2012. This lethal event does not carry the traditional hallmarks of necroptosis or apoptosis (e.g., organelle swelling and chromatin condensation). Instead, cells undergoing ferroptosis have only subtle differences in their morphological features, like smaller-than-normal cell volume and increased mitochondrial membrane density. In this study, we focused on clarifying the role for ferroptosis, an emerging type of cell death, but not the others in AKI.

Ferroptosis may cause changes in the phenotype of TECs. Ferroptosis has been reported to lead to the release of damage-associated molecular patterns and large amounts of lipid peroxides, which may act as interstitial or intercellular messengers [23, 24]. Among all cell types involved in kidney fibrogenesis, macrophages are critical due to their highly flexible programming and activation $[25,26]$. However, the mechanism of macrophage infiltration during ferroptosis is unclear. MCP-1 is one of the most widely studied chemokines in kidney injury [27, 28]. Increased levels of MCP-1 are associated with progressive tubulointerstitial inflammation $[29,30]$, as has been demonstrated in our study. Many signaling pathways can regulate the expression of MCP-1. Previous studies show that Toll-like receptors (TLRs) are widely expressed in TECs [31]. During IRI, sepsis-induced AKI, diabetic nephropathy, and UUO, damage-associated molecular patterns such as high-mobility group boxl protein, histones, and heat-shock proteins released by TECs can activate TLR2 and TLR4 on TECs, resulting in the activation of NF- $\mathrm{KB}$ signaling through MyD88, promoting the release of chemokines such as MCP-1 and other cytokines [32-34]. In UUO nephropathy, upregulation of tubulointerstitial MCP-1 is associated with activating the TGF $\beta / \mathrm{Smad} 3$ signaling pathway $[35,36]$. Besides MCP-1, many other chemokines promote macrophage infiltration. TEC-derived IL-34 also plays a crucial role in aggravating macrophage infiltration and tubular cell injury, leading to persistent 
ischemic AKI and subsequent CKD [37]. CX3CL1 is also detected in renal biopsies from patients with progressive kidney injury [38], and treatment with a neutralizing antibody against CX3CL1 improves renal damage by blocking crescentic formation and macrophage infiltration in rat nephrotoxic nephritis [39]. However, whether there are specific factors released from ferroptotic cells chemoattracting macrophages needs more research. As the most abundant lipid peroxidation product, 4 -HNE has been reported to induce STING carbonylation, thus inhibiting STING activity in macrophages, which may be efficacious in treating autoinflammatory disease [40]. However, its role in renal interstitial inflammation needs further study.

In this study, we demonstrated that inhibiting TEC ferroptosis could reduce kidney fibrosis and interstitial inflammation. Targeting ferroptosis in the kidney may shine a light on ways to protect against kidney fibrosis in patients with CKD.

\section{Statement of Ethics}

All experiments were performed following the approved guidelines and regulations by the Animal Experimentation Ethics Committee at Nanjing Medical University. All animal experimental protocols were approved by Institutional Animal Care and Use Committee (IACUC) at Nanjing Medical University (Approval reference number: IACUC-2103043).

\section{Conflict of Interest Statement}

Chunsun Dai is Associate Editor of the journal of "Kidney Diseases." The authors declare that they have no conflicts of interest with the contents of this article.

\section{Funding Sources}

This work was supported by the National Science Foundation of China Grants 81570611/H0503, 81770675/H0503, and 81770675/H0503; Jiangsu Province's Key Provincial Talents Program ZDRCA2016035; “333” Project (BRA2019101); Six Talents Summit Program (WSN-065) to C. Dai.

\section{Author Contributions}

L. Zhou, X. Xue, and Q. Hou performed the experiments. L. Zhou wrote the manuscript. L. Zhou and X. Xue analyzed the data. C. Dai designed, supervised, and revised the manuscript.

\section{Data Availability Statement}

All data generated or analyzed during this study are included in this article. Further enquiries can be directed to the corresponding author.

\section{References}

1 Chen TK, Knicely DH, Grams ME. Chronic kidney disease diagnosis and management: a review. JAMA. 2019;322(13):1294-304.

2 Breyer MD, Böttinger E, Brosius FC, Coffman TM, Harris RC, Heilig CW, et al. Mouse models of diabetic nephropathy. J Am Soc Nephrol. 2005;16(1):27-45.

3 Kang HM, Ahn SH, Choi P, Ko YA, Han SH, Chinga F, et al. Defective fatty acid oxidation in renal tubular epithelial cells has a key role in kidney fibrosis development. Nat Med. 2015;21(1):37-46.

4 Kirita Y, Wu H, Uchimura K, Wilson PC, Humphreys BD. Cell profiling of mouse acute kidney injury reveals conserved cellular responses to injury. Proc Natl Acad Sci U S A. 2020;117(27):15874-83.

5 Maarouf $\mathrm{OH}$, Aravamudhan A, Rangarajan D, Kusaba T, Zhang V, Welborn J, et al. Paracrine Wnt1 drives interstitial fibrosis without inflammation by tubulointerstitial cross-talk. J Am Soc Nephrol. 2016;27(3):781-90.

6 Humphreys BD. Mechanisms of renal fibrosis. Annu Rev Physiol. 2018;80(1):309-26.
7 Yang L, Brooks CR, Xiao S, Sabbisetti V, Yeung MY, Hsiao LL, et al. KIM-1-mediated phagocytosis reduces acute injury to the kidney. J Clin Invest. 2015;125(4):1620-36.

8 Humphreys BD, Xu F, Sabbisetti V, Grgic I, Naini SM, Wang N, et al. Chronic epithelial kidney injury molecule-1 expression causes murine kidney fibrosis. J Clin Invest. 2013; 123(9):4023-35.

9 Kishi S, Brooks CR, Taguchi K, Ichimura T, Mori Y, Akinfolarin A, et al. Proximal tubule ATR regulates DNA repair to prevent maladaptive renal injury responses. J Clin Invest. 2019;129(11):4797-816.

10 Li H, Peng X, Wang Y, Cao S, Xiong L, Fan J, et al. Atg5-mediated autophagy deficiency in proximal tubules promotes cell cycle G2/M arrest and renal fibrosis. Autophagy. 2016; 12(9):1472-86.

11 Dixon SJ, Lemberg KM, Lamprecht MR, Skouta R, Zaitsev EM, Gleason CE, et al. Ferroptosis: an iron-dependent form of nonapoptotic cell death. Cell. 2012;149(5):106072.
12 Liang C, Zhang X, Yang M, Dong X. Recent progress in ferroptosis inducers for cancer therapy. Adv Mater. 2019;31(51):1904197.

13 Deng F, Sharma I, Dai Y, Yang M, Kanwar YS. Myo-inositol oxygenase expression profile modulates pathogenic ferroptosis in the renal proximal tubule. J Clin Invest. 2019;129(11): 5033-49.

14 Linkermann A, Skouta R, Himmerkus N, Mulay SR, Dewitz C, De Zen F, et al. Synchronized renal tubular cell death involves ferroptosis. Proc Natl Acad Sci U S A. 2014;111(47): 16836-41.

15 Yu Y, Jiang L, Wang H, Shen Z, Cheng Q, Zhang P, et al. Hepatic transferrin plays a role in systemic iron homeostasis and liver ferroptosis. Blood. 2020;136(6):726-39.

16 Liu BC, Tang TT, Lv LL, Lan HY. Renal tubule injury: a driving force toward chronic kidney disease. Kidney Int. 2018;93(3):568-79.

17 Liu BC, Tang TT, Lv LL. How tubular epithelial cell injury contributes to renal fibrosis. Adv Exp Med Biol. 2019;1165:233-52.

18 Chung AC, Lan HY. Chemokines in renal injury. J Am Soc Nephrol. 2011;22(5):802-9. 
19 Sedeek M, Nasrallah R, Touyz RM, Hébert RL. NADPH oxidases, reactive oxygen species, and the kidney: friend and foe. J Am Soc Nephrol. 2013;24(10):1512-8.

20 Prozialeck WC, Edwards JR. Cell adhesion molecules in chemically-induced renal injury. Pharmacol Ther. 2007;114(1):74-93.

21 Geng H, Lan R, Singha PK, Gilchrist A, Weinreb PH, Violette SM, et al. Lysophosphatidic acid increases proximal tubule cell secretion of profibrotic cytokines PDGF-B and CTGF through LPA2- and Gaq-mediated Rho and av $\beta 6$ integrin-dependent activation of TGF- $\beta$. Am J Pathol. 2012;181(4):1236-49.

22 Kobori H, Nangaku M, Navar LG, Nishiyama A. The intrarenal renin-angiotensin system: from physiology to the pathobiology of hypertension and kidney disease. Pharmacol Rev. 2007;59(3):251-87.

23 Sarhan M, von Mässenhausen A, Hugo C, Oberbauer R, Linkermann A. Immunological consequences of kidney cell death. Cell Death Dis. 2018;9(2):114

24 Sarhan M, Land WG, Tonnus W, Hugo CP, Linkermann A. Origin and consequences of necroinflammation. Physiol Rev. 2018;98(2): 727-80.

25 Duffield JS. Cellular and molecular mechanisms in kidney fibrosis. J Clin Invest. 2014; 124(6):2299-306

26 Ricardo SD, van Goor H, Eddy AA. Macrophage diversity in renal injury and repair. J Clin Invest. 2008;118(11):3522-30.
27 Wang Y, Rangan GK, Goodwin B, Tay YC, Harris DC. Lipopolysaccharide-induced MCP-1 gene expression in rat tubular epithelial cells is nuclear factor-kappaB dependent. Kidney Int. 2000;57(5):2011-22.

28 Wang Y, Chen J, Chen L, Tay YC, Rangan GK, Harris DC. Induction of monocyte chemoattractant protein-1 in proximal tubule cells by urinary protein. J Am Soc Nephrol. 1997; 8(10):1537-45.

29 Tesch GH. MCP-1/CCL2: a new diagnostic marker and therapeutic target for progressive renal injury in diabetic nephropathy. Am J Physiol Renal Physiol. 2008;294(4):F697701.

30 Haller H, Bertram A, Nadrowitz F, Menne J. Monocyte chemoattractant protein-1 and the kidney. Curr Opin Nephrol Hypertens. 2016; 25(1):42-9.

31 Leemans JC, Kors L, Anders HJ, Florquin S. Pattern recognition receptors and the inflammasome in kidney disease. Nat Rev Nephrol. 2014;10(7):398-414.

32 Allam R, Scherbaum CR, Darisipudi MN Mulay SR, Hägele H, Lichtnekert J, et al. Histones from dying renal cells aggravate kidney injury via TLR2 and TLR4. J Am Soc Nephrol. 2012;23(8):1375-88.

33 Wu H, Ma J, Wang P, Corpuz TM, Panchapakesan U, Wyburn KR, et al. HMGB1 contributes to kidney ischemia reperfusion injury. J Am Soc Nephrol. 2010;21(11):187890.

34 Leemans JC, Stokman G, Claessen N, Rouschop KM, Teske GJ, Kirschning CJ, et al. Renal-associated TLR2 mediates ischemia/reperfusion injury in the kidney. J Clin Invest. 2005;115(10):2894-903.
35 Chung AC, Huang XR, Zhou L, Heuchel R, Lai KN, Lan HY. Disruption of the Smad7 gene promotes renal fibrosis and inflammation in unilateral ureteral obstruction (UUO) in mice. Nephrol Dial Transplant. 2009;24(5): 1443-54.

36 Ji X, Wang H, Wu Z, Zhong X, Zhu M, Zhang Y, et al. Specific inhibitor of Smad3 (SIS3) attenuates fibrosis, apoptosis, and inflammation in unilateral ureteral obstruction kidneys by inhibition of transforming growth factor beta (TGF-beta)/Smad3 signaling. Med Sci Monit. 2018;24:1633-41.

37 Baek JH, Zeng R, Weinmann-Menke J, Valerius MT, Wada Y, Ajay AK, et al. IL-34 mediates acute kidney injury and worsens subsequent chronic kidney disease. J Clin Invest. 2015;125(8):3198-214.

38 Koziolek MJ, Schmid H, Cohen CD, Blaschke S, Hemmerlein B, Zapf A, et al. Potential role of fractalkine receptor expression in human renal fibrogenesis. Kidney Int. 2007;72(5): 599-607.

39 Yoshimoto S, Nakatani K, Iwano M, Asai O, Samejima K, Sakan H, et al. Elevated levels of fractalkine expression and accumulation of CD16+ monocytes in glomeruli of active lupus nephritis. Am J Kidney Dis. 2007;50(1): 47-58.

40 Jia M, Qin D, Zhao C, Chai L, Yu Z, Wang W, et al. Redox homeostasis maintained by GPX4 facilitates STING activation. Nat Immunol. 2020;21(7):727-35. 